\title{
Preventing Hydrogen Disposal Increases Electrode Utilization Efficiency by Shewanella oneidensis
}

\section{OPEN ACCESS}

Edited by:

Daniel Puyol,

Rey Juan Carlos University, Spain

Reviewed by:

Jung Rae Kim,

Pusan National University,

South Korea

Falk Harnisch,

Helmholtz Centre for Environmental

Research (UFZ), Germany

*Correspondence:

Jeffrey A. Gralnick

gralnick@umn.edu

Daniel R. Bond

dbond@umn.edu

tThese authors have contributed equally to this work

Specialty section:

This article was submitted to Bioenergy and Biofuels,

a section of the journal

Frontiers in Energy Research

Received: 16 June 2019

Accepted: 26 August 2019 Published: 11 September 2019

Citation:

Joshi K, Kane AL, Kotloski NJ,

Gralnick JA and Bond DR (2019)

Preventing Hydrogen Disposal

Increases Electrode Utilization Efficiency by Shewanella oneidensis.

Front. Energy Res. 7:95.

doi: 10.3389/fenrg.2019.00095

\author{
Komal Joshi ${ }^{1 \dagger}$, Aunica L. Kane ${ }^{1 \dagger}$, Nicholas J. Kotloski ${ }^{1}$, Jeffrey A. Gralnick ${ }^{1,2 *}$ and \\ Daniel R. Bond ${ }^{1,2 *}$
}

${ }^{1}$ BioTechnology Institute, University of Minnesota-Twin Cities, St. Paul, MN, United States, ${ }^{2}$ Department of Plant and Microbial Biology, University of Minnesota-Twin Cities, St. Paul, MN, United States

Many bacteria use hydrogen anaerobically as both a source and sink for electrons; consuming hydrogen when it is plentiful and producing it when concentrations are low enough to allow proton reduction. While this can increase an organism's competitiveness, hydrogen uptake, or excretion can also make it difficult to control electron flux to a specific product. For example, when Shewanella oneidensis strain MR-1 is used to oxidize organic molecules and recover electrons in microbial electrochemical devices, small changes in ambient hydrogen concentrations could dramatically alter the efficiency of electron capture at the anode due to this organism's respiratory flexibility. When new three-electrode reactor designs created to minimize oxygen intrusion during anodic growth were tested with lactate-oxidizing $S$. oneidensis, current production decreased significantly in reactors vented to remove hydrogen produced at the counter electrode, suggesting a role for hydrogen uptake or disposal when cells used electrodes as electron acceptors. A $\Delta$ hydA $\Delta$ hyaB mutant lacking both hydrogenases reversed this trend, and nearly doubled current production rates. This increase was shown to be due to the efficiency of lactate oxidation, as $90 \%$ of electrons were recovered as electricity in the $\triangle$ hydA $\triangle$ hyaB mutant compared to only $50 \%$ for wild type. Experiments with $\mathrm{Fe}$ (III) oxide provided additional evidence that $S$. oneidensis generates hydrogen reducing equivalents during reduction of insoluble electron acceptors, while experiments with cells incubated with Fe(III) citrate showed increased survival of wild-type compared to $\Delta h y d A \Delta h y a B$ in stationary phase. Together these data show how the multiple routes of electron disposal of S. oneidensis, while beneficial under changing conditions, limits the efficiency of electron recovery in electrochemical systems, and demonstrates a simple approach to increasing current production rates in systems where hydrogen is being captured as a product.

Keywords: extracellular electron transfer, hydrogen metabolism, coulombic efficiency, hydrogenase, Shewanella, microbial fuel cells

\section{INTRODUCTION}

Life began, and continues to thrive today, in anaerobic ecosystems across the planet. A variety of metabolic strategies allow life to persist in anaerobic conditions within environments such as sediments, subsurface aquifers, stratified lakes, digestive tracts, wastewater treatment plants, and anaerobic digesters. For heterotrophic respiratory organisms, life under anaerobic conditions 
is often constrained by the ability of cells to dispose of electrons generated during carbon compound oxidation; thus the availability of electron acceptors such as nitrate or Fe(III) controls the options for energy generation within a niche (Thauer et al., 1977). In all of these environments, hydrogenases represent a means to dispose of low-potential electrons via proton reduction, depending on ambient concentrations. Here, we explore the effect of this hydrogen disposal choice in a dissimilatory metal reducing bacterium, and show that while hydrogen production may benefit an organism in terms of electron balance, it significantly decreases the efficiency of electricity-generating devices due to diversion of electron flux away from the electrode.

Shewanella oneidensis strain MR-1 (hereafter referred to as MR-1) is a facultative anaerobe that thrives in redox-stratified environments due to its ability to utilize a wide range of terminal electron acceptors (Nealson and Scott, 2006; Hau and Gralnick, 2007). MR-1 is able to couple the oxidation of electron donors such as lactate, formate, and hydrogen, to reduction of over 20 organic and inorganic compounds including insoluble metal oxides (Nealson and Scott, 2006; Hau and Gralnick, 2007; Kane et al., 2016). The process of transferring electrons produced during anaerobic metabolism to insoluble terminal electron acceptors is termed extracellular electron transfer, and has been well-studied in MR-1 (Beblawy et al., 2018). During extracellular electron transfer, electrons flow from the quinone pool through a series of cytochromes known as the Mtr respiratory pathway to the exterior of the cell, where they are transferred to solid acceptors directly or with the aid of secreted flavin-based redox shuttles (Beblawy et al., 2018). The ability of MR-1 to couple organic carbon oxidation to reduction of insoluble metals is important in biogeochemical cycling, and in applications where electrodes within bioelectrochemical reactors serve as proxies for the metals used as terminal electron acceptors (Rabaey and Verstraete, 2005; Bretschger et al., 2007; Hau and Gralnick, 2007; Fredrickson et al., 2008; Coursolle et al., 2010). For example, when Shewanella is used to supply electrons to the anode in a device termed a "Microbial Electrolysis Cell," a small voltage can be applied to these electrons to produce valuable cathodic hydrogen at a counter electrode (Bretschger et al., 2007; Coursolle et al., 2010).

To better harness extracellular electron transfer in bioenergy and bioelectronic applications, knowing the fate of electrons generated during metabolic oxidation is essential. Coulombic efficiency is calculated by comparing the number of electrons released during oxidation of the donor to the electron equivalents recovered at the anode. Efficiency decreases when electrons are diverted to products or acceptors other than the anode. For example, experimental artifacts such as leakage of oxygen into electrode chambers in a two chambered bioreactor containing Shewanella supports higher cell density and synthesis of higher concentrations of flavins that enhance electron transfer, but at the expense of decreasing coulombic yield due to oxygen respiration and formation of biomass (Rosenbaum et al., 2010; TerAvest et al., 2014; Lu et al., 2017). Inefficient transfer of protons across chambers from membrane fouling as well leaking of substrates or oxygen from the cathode chamber into the anode chamber results in low coulombic efficiency. In single chamber devices where a counter electrode produces hydrogen, coulombic efficiency can appear to exceed $100 \%$ if cells re-uptake produced hydrogen, a process that wastes energy by applying energy to the same electron equivalents multiple times (Lee et al., 2009; Lee and Rittmann, 2010). Conversely, MR-1 can also produce hydrogen during lactate oxidation, representing a loss of energy and electrons (Pinchuk et al., 2011). Thus, due to the high affinity of MR-1 for oxygen, and the reversible nature of hydrogenases, very small alterations in experimental design can cause large changes in the performance of microbial electrochemical systems (Lee et al., 2009; Lee and Rittmann, 2010). It should be noted that these challenges are accentuated in single chamber three electrode bioreactors where a single organism is used. Hydrogen dynamics will differ substantially in membrane-separated systems and in mixed-culture systems.

The genome of MR-1 contains two hydrogenase gene clusters that contribute to hydrogen production and uptake. The cluster hyaABCDE-hypF-hypBCDEA (SO2089-2099) encodes a Group 1 [NiFe] hydrogenase and the hydrogenase pleitropy (hyp) maturation proteins, while hydAB-fdhC-hydGXEF (SO39203926) encodes an $[\mathrm{FeFe}]$ hydrogenase, maturation proteins, and a gene annotated as a formate dehydrogenase gamma subunit (Meshulam-Simon et al., 2007). The HydA hydrogenase functions primarily in hydrogen formation while $\mathrm{HyaB}$ is capable both of hydrogen production and uptake (MeshulamSimon et al., 2007). Both hydrogenases are expressed during anaerobic growth, and hydrogen production has been observed in cultures following depletion of the electron acceptor fumarate (Meshulam-Simon et al., 2007; Kreuzer et al., 2014).

Here, we describe new electrochemical reactors capable of maintaining strictly anaerobic conditions to eliminate artifacts from aerobic growth, combined with modifications that prevent hydrogen recycling. These reactors allow investigation of the role of hydrogen and the $\mathrm{HydA} / \mathrm{HyaB}$ systems in altering coulombic efficiency of bioelectrochemical reactions, revealing new insights into the anaerobic metabolic strategy of Shewanella. We demonstrate that deletion of hydA and hyaB in Shewanella results in higher current density and coulombic efficiency by diverting electron flux to the anode instead of to hydrogen disposal. Hydrogenase-containing cells are shown to produce a burst of reducing equivalents when exposed to another insoluble electron acceptor (Fe(III) oxide), and the presence of hydrogenases enhances survival when other electron donors are depleted during growth with $\mathrm{Fe}(\mathrm{III})$ citrate. This evidence suggests that hydrogen production provides S. oneidensis with an electron disposal mechanism, and that eliminating this ability to use protons as electron acceptors diverts oxidative metabolism to exclusive use of the Mtr respiratory pathway, allowing enhanced capture of electrons in electrochemical cells.

\section{MATERIALS AND METHODS}

\section{Bacterial Strains and Culture Conditions}

Strains and plasmids used in this study are listed in Table 1. S. oneidensis MR-1 has been described previously (Venkateswaran et al., 1999). Escherichia coli strains used for cloning (UQ950) and conjugal transfer (WM3064) were 
TABLE 1 | Strains and plasmid used in this study.

\begin{tabular}{lll}
\hline Strain or plasmid & Characteristics & Reference/Source \\
\hline $\begin{array}{l}\text { S. oneidensis } \\
\text { strain MR-1 }\end{array}$ & Wild-type & $\begin{array}{l}\text { Venkateswaran et al., } \\
1999\end{array}$ \\
$\Delta$ hydA $h$ hya & S. oneidensis $\triangle$ hydA $\Delta$ hyaB & This study \\
E. coli WM3064 & DAP auxotroph E. coli conjugal & Saltikov and Newman, \\
& donor strain & 2003 \\
E. coli UQ950 & DH5 $\alpha$; cloning strain & Saltikov and Newman, \\
& & 2003 \\
pSMV3 & $9.1 \mathrm{~kb}$ suicide vector; oriR6K, & Saltikov and Newman, \\
& mobRP4, sacB, Km & 2003 \\
& &
\end{tabular}

maintained on lysogeny broth (LB) agar plates containing $50 \mu \mathrm{g} / \mathrm{mL}$ kanamycin and/or $250 \mu \mathrm{M}$ 2,6-diaminopimelic acid as necessary at $37^{\circ} \mathrm{C}$ and have been described previously (Saltikov and Newman, 2003). S. oneidensis strains were maintained on LB agar plates at $30^{\circ} \mathrm{C}$. Shewanella basal media (SBM) pH 7.2 was used where indicated and contained the following (per liter): $0.225 \mathrm{~g} \mathrm{~K}_{2} \mathrm{HPO}_{4}, 0.225 \mathrm{~g} \mathrm{KH}_{2} \mathrm{PO}_{4}, 0.46 \mathrm{~g} \mathrm{NaCl}, 0.225 \mathrm{~g}$ $\left(\mathrm{NH}_{4}\right)_{2} \mathrm{SO}_{4}, 0.117 \mathrm{~g} \mathrm{MgSO}_{4} .7 \mathrm{H}_{2} \mathrm{O}, 5 \mathrm{~mL}$ vitamin mix, $5 \mathrm{ml}$ of trace mineral mix, and $0.05 \%$ casamino acids (Hau et al., 2008). For growth assays, $S$. oneidensis strains were freshly streaked from $-80^{\circ} \mathrm{C}$ glycerol stocks on $\mathrm{LB}$ plates and incubated for $\sim 16 \mathrm{~h}$ at $30^{\circ} \mathrm{C}$. Single colonies from these plates were used to inoculate $5 \mathrm{~mL}$ of liquid LB medium shaken at $250 \mathrm{rpm}$ at $30^{\circ} \mathrm{C}$ for $8 \mathrm{~h}$ and were then sub-cultured into $5 \mathrm{~mL}$ of SBM supplemented with $20 \mathrm{mM}$ lactate for $\sim 16 \mathrm{~h}$ shaken at $250 \mathrm{rpm}$ at $30^{\circ} \mathrm{C}$. Cultures were then washed twice and added to SBM containing $20 \mathrm{mM}$ lactate and $40 \mathrm{mM}$ fumarate in tubes sealed with butyl rubber stoppers containing an argon headspace.

\section{Reagents and Materials}

Enzymes were purchased from New England Biolabs (Ipswich, MA). Kits for gel and plasmid purification were purchased from Invitrogen (Carlsbad, CA). All related reactions were carried out according to manufacturer instructions. Media components were purchased from Becton, Dickinson and Company (Sparks, $\mathrm{MD}$ ), and chemicals including sodium DL-lactate and disodium fumarate were purchased from Sigma-Aldrich (St. Louis, MO).

\section{Generation of Deletion Mutants}

Oligonucleotide primers used to amplify regions of the MR-1 chromosome for deletion constructs are listed in Table 2. Regions of $\sim 500$ base pairs both up and downstream of the target deletion region were amplified using primers hyaB-UF/hyaB-UR, hyaBDF/hyaB-DR, hydA-UF/hydA-UR, and hydA-DF/hydA-DR with high fidelity polymerase Pfu Ultra (Agilent Technologies, Santa Clara, CA). Following restriction digest, up and downstream fragments were gel purified and cloned into the suicide vector pSMV3. Deletion constructs were introduced into MR-1 via conjugal transfer using E. coli donor strain WM3064 (Saltikov and Newman, 2003). In-frame gene deletions for hydA and hyaB were generated by homologous recombination as described previously (Saltikov and Newman, 2003). Plasmid constructs
TABLE 2 | Primers used in this study.

\begin{tabular}{|c|c|}
\hline Primer name & Sequence \\
\hline \multicolumn{2}{|c|}{ Primers for hydA deletion: } \\
\hline hydA-UF & GCATGGGCCCGCATTATCAATTCACCATAAACCC \\
\hline hydA-UR & GCATACTAGTATTAATCTTGATCAGCCC \\
\hline hydA-DF & GCATACTAGTGTGAAATCAGCCTCTGTC \\
\hline hydA-DR & GCATGAGCTCTITGCTAGGCTGTCGTCCTTG \\
\hline \multicolumn{2}{|c|}{ Primers for hyaB deletion: } \\
\hline hyaB-UF & GCATGGGCCCGTGGCCGTITGATGCAG \\
\hline hyaB-UR & GCATACTAGTITCAGTATGACTTCAATAAC \\
\hline hyaB-DF & GCATACTAGTGATGCTGTCAATGCCCTG \\
\hline hyaB-DR & GCATGAGCTCATGCGGGTTCAGAATGG \\
\hline \multicolumn{2}{|c|}{ Primers for sequencing: } \\
\hline hydA-SP1 & TTCGACTCTACCTATGAAGCAATTAC \\
\hline hydA-SP2 & GATGTGCACATCATAGGTTAGCTG \\
\hline hyaB-SP1 & GTCAGCAAACCCGTGATTAACTTAG \\
\hline hyaB-SP2 & GATCCAACCTTGTACTAATACATCCGT \\
\hline
\end{tabular}

and deletion strains were sequence verified using primers hyaBSP1/hyaB-SP-2 and hydA-SP1/hydA-SP2 at the University of Minnesota Genomics Center.

\section{Anaerobic Bioreactors Vented to Remove Hydrogen}

To eliminate intrusion of oxygen into bioelectrochemical reactors, a new sealed top mechanism was machined for $25 \mathrm{~mL}$ glass cones (Bioanalytical Systems, West Lafayette, IN), from PEEK (a polyether ether ketone thermoplastic polymer). PEEK is gas impermeable and mechanically robust, supporting a compression seal around openings and threaded ports for stainless steel fittings (McMaster-Carr). The bioreactor top has three ports at the center to house three electrodes (working, counter, and salt bridge for a reference electrode). The electrodes were fed through the top with the help of gaskets and a PEEK disc compressed by a threaded screw at the center (Figure 1A). Tightening of the center screw compresses the gasket, providing a seal on the glass tubing used for electrodes. Stainless steel screws mounted around the outer edge pull a ring from below, compressing the top of the reactor in a gasket within a groove. This design ensures that the distance between electrodes and the reference relative to the working and counter stays constant in every experiment, decreasing variability and increasing repeatability. Supplementary Figure 1 is an overview of the reactor design, with detailed CAD file drawings and parts description list available here: https://github.com/komaljoshiumn/Bond_lab_reactors.

At all stages of design and testing, oxygen levels were measured using a Series 3,000 Trace Oxygen Analyzer capable of detecting $\mathrm{O}_{2}$ levels in the gas phase below 1 ppm (Alpha Omega, Lincoln RI). Anoxic conditions were maintained by slowly flushing reactors with argon purified of oxygen using a heated copper column, and oxygen in the headspace was measured to be below $2 \mathrm{ppm}$ even at $<1 \mathrm{ml} / \mathrm{min}$ flushing rate. 
A

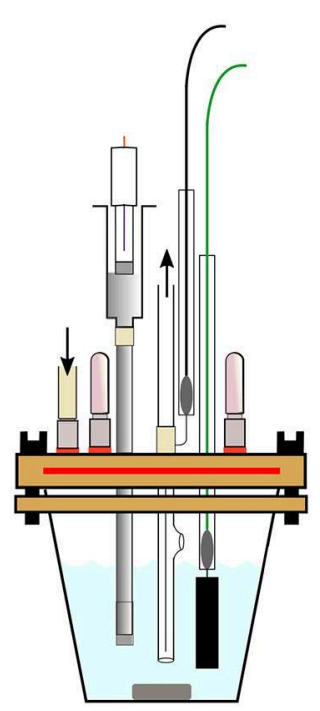

B

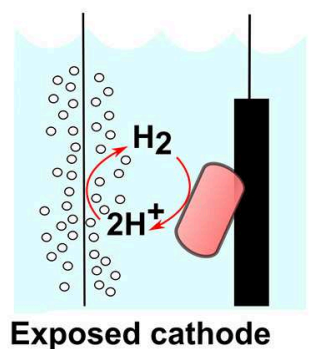

Vented cathode
C

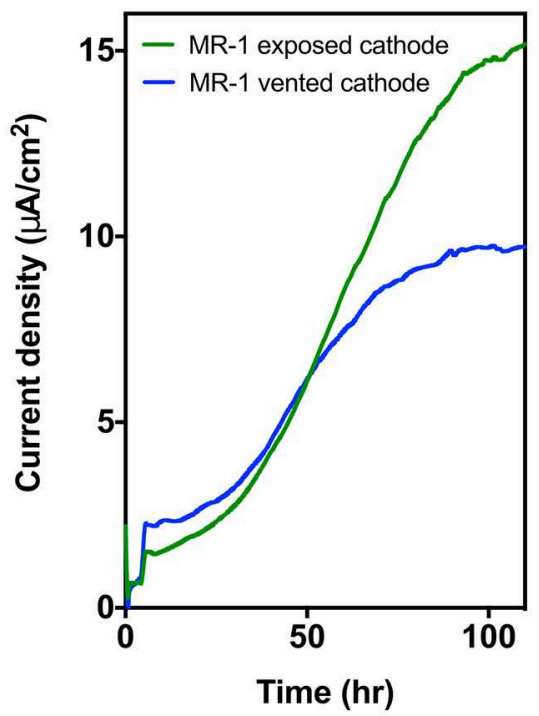

FIGURE 1 | MR-1 produces lower current density when cathodic hydrogen is vented. (A) Model of the new strictly anaerobic three electrode electrochemical cell. (B) Designs for exposed vs. vented cathodes to control release of cathodic hydrogen into the reactor. Hydrogen from the exposed cathode could suppress hydrogen disposal by bacteria, or serve as an electron donor if concentrations are high enough. (C) Current density over time for MR-1 cultures in new bioreactors containing exposed (green) and vented (blue) cathodes anodes poised at $+0.240 \mathrm{~V}$ vs. SHE. Representative data from three independent experiments $(n=3)$ are shown.

Argon was bubbled through sealed vessels containing anoxic MilliQ water for humidification. When needed, flexible tubing for making connections was fresh Pharmed BPT tubing (Cole Parmer, Vernon Hills IL), as we found that after exposure to multiple $(>5)$ rounds of autoclaving, $0.5 \mathrm{~m}$ of flexible tubing allowed in as much as $5 \mathrm{ppm} \mathrm{O}_{2}$. The working electrode consisted of a graphitic carbon electrode measuring $0.5 \mathrm{~cm} \times$ $3 \mathrm{~cm} \times 1 \mathrm{~mm}$ connected to a platinum wire with a Teflon bolt (McMaster Carr, Elmhurst, IL). The working electrode was polished with 400 grit sandpaper, rinsed in $1 \mathrm{~N} \mathrm{HCl}$ and cleaned by sonication in deionized water twice for $10 \mathrm{~min}$ prior to use. The reference consisted of a glass body $\mathrm{Ag} / \mathrm{AgCl}$ electrode in $3 \mathrm{M}$ $\mathrm{KCl}$ connected to the system via a glass capillary tube filled with $1 \%$ agarose in a $0.1 \mathrm{M} \mathrm{KCl}$ solution and capped with a vycor frit. The counter electrode consisted of a platinum wire, and when indicated was contained within glass tubing that was vented from the reactor to eliminate introduction of hydrogen into the system. Bioreactors were maintained at $30^{\circ} \mathrm{C}$ using a water bath and were stirred continuously. Electrochemical data was monitored using a 16-channel VMP ${ }^{\circledR}$ potentiostat (Bio-Logic, Claix, France).

\section{Bioreactor Preparation and Electrochemical Analysis}

For bioreactor assays, single $S$. oneidensis colonies were used to inoculate $\mathrm{LB}$ medium incubated at $30^{\circ} \mathrm{C}, 250 \mathrm{rpm}$ for $\sim 16 \mathrm{~h}$. Strains were then sub-cultured in LB supplemented with $20 \mathrm{mM}$ lactate and $40 \mathrm{mM}$ fumarate under an argon headspace. When anaerobic LB cultures reached an optical density measured at $600 \mathrm{~nm}\left(\mathrm{OD}_{600}\right)$ of $0.5-0.6,1 \mathrm{~mL}$ of culture was added to bioreactors containing $14 \mathrm{~mL}$ of SBM medium containing $50 \mathrm{mM}$ $\mathrm{NaCl}, 100 \mathrm{mM}$ HEPES buffer, and $60 \mathrm{mM}$ lactate, and $40 \mathrm{mM}$ fumarate. After $\sim 5 \mathrm{~h}$, the fumarate acceptor in these cultures is exhausted, and cells begin respiring electrodes (reflected in sudden jumps in current in Figures 1, 2). Following inoculation, the working electrode was poised at $+0.240 \mathrm{~V}$ vs. SHE (Standard Hydrogen Electrode), and current was monitored for $\sim 120 \mathrm{~h}$ until current plateaued. Reactors were then washed three times with SBM containing $50 \mathrm{mM} \mathrm{NaCl}$ but lacking electron donor or acceptor, and current was monitored until basal levels were reached indicating depletion of lactate. SBM containing $50 \mathrm{mM}$ $\mathrm{NaCl}, 30 \mathrm{mM}$ lactate, $1 \mu \mathrm{M}$ riboflavin, and $10 \mathrm{mM}$ arabinose (arabinose included as an internal HPLC standard not utilized as a carbon source by MR-1) was then added to reactors and current analysis was continued for $96 \mathrm{~h}$ (Rodionov et al., 2010; Golitsch et al., 2013). Supernatant was removed every $24 \mathrm{~h}$ for metabolite analysis by HPLC.

\section{High Performance Liquid Chromatography (HPLC) and Coulombic Efficiency Calculations}

Metabolites were quantified by HPLC using Shimadzu Scientific equipment including an SCL-10A system controller, LC-10AT 


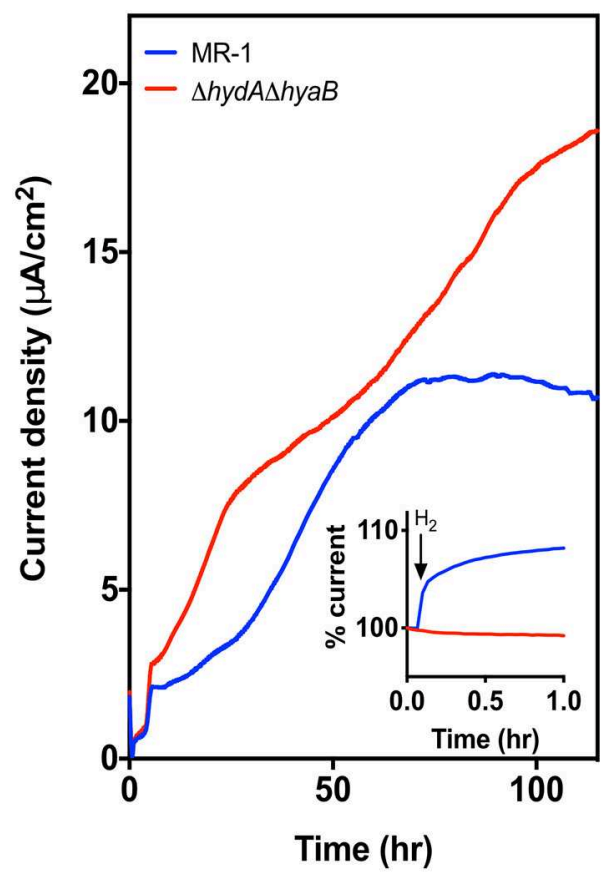

FIGURE 2 | Deletion of hydrogenases results in higher current density. Current density produced by MR-1 (blue) vs. $\triangle h y d A \Delta h y a B$ (red) in anaerobic bioreactors with vented cathodes. Lactate was supplied as the carbon source, and graphite carbon anodes were poised at $+0.240 \mathrm{~V}$ vs. SHE. Data represents average current density produced in reactors run in triplicate. Inset figure shows the response in current production to addition of $1 \%$ exogenous hydrogen into the reactor headspace, with level of current at time of injection represented as $100 \%$.

pump, SIL-10AF auto-injector, CTO-10A column oven, RID$10 \mathrm{~A}$ refractive index detector $(210 \mathrm{~nm})$, and an SPD-10A UVVis detector. Mobile phase consisted of $15 \mathrm{mN} \mathrm{H}_{2} \mathrm{SO}_{4}$ set at

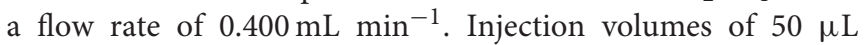
were separated on an Aminex HPX-87H column maintained at $46^{\circ} \mathrm{C}$. Reactor samples were compared to standard curves generated from known concentrations of each metabolite $\left(R^{2}>\right.$ 0.99). These data were used to calculate coulombic efficiency, as Lactate $\rightarrow$ Acetate $+\mathrm{CO}_{2}+4 \mathrm{e}^{-}$, and Coulombic efficiency $(\%)=($ moles of electrons collected at the electrode) / (moles of electrons recovered as acetate $\times 4)$.

\section{Fe(III) Oxide Reduction}

Strains were streaked on LB agar plates from $-80^{\circ} \mathrm{C}$ stocks at $30^{\circ} \mathrm{C}$ incubator overnight. Single colonies were inoculated into $\mathrm{LB}$ medium incubated at $30^{\circ} \mathrm{C}, 250 \mathrm{rpm}$ for $\sim 16 \mathrm{~h}$. Strains were then sub-cultured in anaerobic LB medium containing $20 \mathrm{mM}$ lactate and $40 \mathrm{mM}$ fumarate under anaerobic conditions. After the cultures reached an $\mathrm{OD}_{600 \mathrm{~nm}}$ of $0.5-0.55,1 \mathrm{~mL}$ of culture was inoculated into bioreactors containing $14 \mathrm{~mL}$ of SBM with $50 \mathrm{mM}$ Fe(III) oxide, and $10 \mathrm{mM}$ lactate. Platinum redox probes were included in the bioreactors to continuously measure the redox potential of the system. Bioreactors were maintained at $30^{\circ} \mathrm{C}$ using a water bath and were stirred continuously. Change in redox potential over time was measured using OCV (open circuit voltage) technique using a 16-channel VMP ${ }^{\circledR}$ potentiostat (Bio-Logic). Calibration redox probe measurements were also performed in the presence of Fe(III) oxide, except no cells were added and $10-1 \%$ hydrogen was added in the headspace.

\section{Fe(III) Citrate Reduction Assay}

Strains were streaked on LB agar plates from $-80^{\circ} \mathrm{C}$ stocks as described for $\mathrm{Fe}(\mathrm{III})$ oxide experiments, and after subcultures reached an $\mathrm{OD}_{600 \mathrm{~nm}}$ of $0.5-0.55,1 \mathrm{~mL}$ of culture was inoculated into $14 \mathrm{~mL}$ of SBM with $50 \mathrm{mM} \mathrm{Fe}$ (III) citrate and $10 \mathrm{mM}$ lactate. Following inoculation, $200 \mu \mathrm{L}$ samples were taken using a degassed syringe at regular time intervals. Hundred microliter of sample was saved into $900 \mu \mathrm{L}$ of $0.5 \mathrm{~N} \mathrm{HCl}$ for the FerroZine assay and the remaining $100 \mu \mathrm{L}$ was used to determine cell density. Fe(II) was measured in the samples using a modified FerroZine assay (Lovley and Phillips, 1987).

To determine cell density during Fe citrate reduction, 100 $\mu \mathrm{L}$ samples were removed at the time points indicated using a degassed syringe. Samples were then serially diluted, plated on LB agar, and colonies were counted after incubation overnight at $30^{\circ} \mathrm{C}$.

\section{RESULTS}

Using a three-electrode potentiostat-poised system, a working electrode can be maintained at a constant potential, and respiration by bacteria capable of extracellular electron transfer can be monitored in real-time. In this case, the graphite electrode was poised at $+0.24 \mathrm{~V}$ vs. SHE, allowing bacteria to oxidize lactate and utilize the anode as a terminal electron acceptor (Baron et al., 2009). Electron transfer efficiency to the electrode can be influenced due to oxygen acting as an alternative electron acceptor, by hydrogen produced by cells escaping into the headspace, or by hydrogen evolved from the counter electrode (cathode) serving as an additional electron donor. The design shown in Figure 1A (for detailed design, see Supplementary Figure 1) was optimized to eliminate all routes for oxygen entry, which then resulted in a device so well-sealed that gases produced in the medium could remain within the reactor (Figure 1B). To then isolate the effect of hydrogen, we modified reactors further by containing counter electrodes within tubing that vented produced hydrogen from the chamber, and compared the current density of strictly anaerobic MR-1 utilizing electrodes in vented vs. exposed designs.

\section{Venting Cathodic Hydrogen Decreases Current Production by MR-1 in Three-Electrode Bioreactors}

Cathodic hydrogen produced at exposed counter electrodes can raise hydrogen levels to concentrations that thermodynamically prevents hydrogen production by cells. At higher levels, hydrogen can act as an additional electron donor (Geelhoed and Stams, 2011; Schink and Stams, 2013) (Figures 1A,B). When cathodic hydrogen was vented from oxygen-free reactors operated in triplicate for each condition, current density by 
MR-1 was significantly diminished compared to reactors with exposed cathodes (Figure 1C). Venting hydrogen out of the reactors decreased current density by $36.6 \pm 4.5 \%$, suggesting either that hydrogen inhibited electron flow out of MR-1, or that a significant portion of current density resulted from re-utilization of hydrogen (Figure 1C). To investigate this metabolic choice, mutants unable to produce or use hydrogen were constructed.

\section{Hydrogenase Mutants Produce Higher Current Density in Single-Chamber, Three-Electrode Bioreactors}

Electrons produced during anaerobic metabolism can either be transported from the quinone pool to external acceptors via the Mtr pathway, or, if their redox potential is low enough relative to the ambient hydrogen concentration, shunted to hydrogenases (Pinchuk et al., 2011). To quantify the role of hydrogen metabolism, marker-less in-frame deletions were constructed in the regions encoding both large subunits of the hydrogenases in the MR-1 genome (strain referred to as $\triangle h y d A \Delta h y a B$ ). Deletion of both hydrogenases did not adversely affect growth rate or yield of $\triangle h y d A \triangle h y a B$ utilizing lactate as the donor and fumarate as the acceptor (Supplementary Figure 2).

In contrast, when triplicate MR-1 or $\triangle h y d A \Delta h y a B$ cultures were grown with lactate in anaerobic bioreactors with vented cathodes, maximum current densities for $\triangle h y d A \Delta h y a B$ were significantly higher compared to wild type $(p$-value $<0.0001)$ (Figure 2). The rate of current increase was also faster for all $\triangle h y d A \triangle h y a B$ reactors during the first $20 \mathrm{~h}$ following inoculation, and continued to increase for $60 \mathrm{~h}$. MR-1 produced very little current over the first $20 \mathrm{~h}$, and plateaued much sooner (Figure 2).

As the $\triangle h y d A \triangle h y a B$ mutant lacks options for electron flux out of the cell via hydrogenases, this improvement was consistent with a strict dependence on using the electrode. While MR-1 can only oxidize lactate to acetate under anaerobic conditions, under low cell density conditions in reactors lacking hydrogen, MR-1 could dispose of some electrons via hydrogen production and not be as dependent upon the electrode as its sole electron acceptor. To verify the presence or absence of hydrogenases under these conditions, $1 \%$ hydrogen was injected into the reactor headspace, causing current production to increase in MR1 cultures for over $5 \mathrm{~h}$, while no such increase was seen in mutant cultures (Figure 2, inset). This illustrated how sensitive MR-1 current production is to ambient hydrogen concentrations, and confirmed that the $\triangle h y d A \triangle h y a B$ mutant was insensitive to the presence of hydrogen.

\section{Deletion of Hydrogenases Increases Efficiency of Electron Capture in Three-Electrode Bioreactors}

After cultivating both strains for $120 \mathrm{~h}$ (Figure 2), all replicates of wild type and mutant cultures reached their maximum current density, with $\triangle h y d A \Delta h y a B$ cultures always producing more current than wild type. To test whether this increased current was due to diversion of more electrons per lactate to the electrode, the reactor medium was exchanged with fresh medium containing $30 \mathrm{mM}$ lactate, $1 \mu \mathrm{M}$ riboflavin, and $10 \mathrm{mM}$ arabinose (included as an internal control for HPLC). Following medium replacement, $\triangle h y d A \Delta h y a B$ cultures sustained higher current densities, demonstrating nearly twice the rate of electron transfer to the anode as that of MR-1, and the attached biofilms maintained this constant plateau for over 4 days (Figure 3A, $n=3$ for each strain). Samples were removed from triplicate reactors during these incubations and analyzed by HPLC to determine the fate of the lactate donor, which S. oneidensis typically oxidizes to acetate and four electrons under anaerobic conditions (Hunt et al., 2010; Pinchuk et al., 2011).

While $\triangle h y d A \Delta h y a B$ biofilms produced current nearly twice as fast as MR-1, acetate production rates from lactate were not as significantly different between the two strains: $\triangle$ hydA $\triangle$ hyaB produced $42 \pm 3 \mu \mathrm{M}$ acetate $\mathrm{hr}^{-1}$, while MR-1 produced $38 \pm 3 \mu \mathrm{M}$ acetate $\mathrm{hr}^{-1}$ (Figure 3B). This was consistent with each culture consuming lactate at similar rates, while $\triangle h y d A \triangle h y a B$ biofilms diverted significantly more electrons to electrodes. When coulombic efficiencies were calculated by dividing the moles of electrons recovered at electrodes by the moles of lactate oxidized to acetate, nearly $90 \%$ of electrons were recovered as electricity in the $\triangle$ hydA $\Delta h y a B$ strain, while this ratio was only $52 \%$ for MR- 1 under the same conditions (Figure 3C). Based on these results, when hydrogen concentrations are low, MR-1 can dispose of nearly half of its electrons via hydrogen production.

\section{Evidence for Hydrogen Disposal During Reduction of Fe(III) Oxide}

Shewanella cells need time to attach to solid acceptors such as $\mathrm{Fe}$ (III) oxides or electrodes, and electron transfer to these acceptors is accelerated by soluble shuttles. Thus, cells exposed to insoluble extracellular acceptors experience an excess of electrons. Hydrogen production should also be detectable in cultures exposed to metal oxides, if they represent a sustained bottleneck to electron disposal similar to what is observed with electrode-based acceptors.

For these experiments, MR-1 and the $\triangle h y d A \Delta h y a B$ strain were inoculated into medium with only particulate $\mathrm{Fe}(\mathrm{III})$ oxide as the electron acceptor, and a platinum redox probe was included to detect hydrogen. When MR-1 and the $\Delta$ hydA $\Delta$ hyaB were inoculated into $\mathrm{Fe}$ (III) oxide medium, the redox potential dropped in MR-1 cultures immediately after introduction of cells, while this change did not occur in $\triangle h y d A \Delta h y a B$ (Figure 4A). Very little $\mathrm{Fe}$ (II) was detected at this early stage (data not shown), suggesting that wild type cells experiencing excess donor and limited ability to access Fe(III) oxide as the acceptor produced a burst of hydrogen to dispose of excess electrons.

In control experiments, when hydrogen gas was added directly into the headspace of chambers containing Fe(III) oxide medium alone, a near instantaneous drop in redox potential was also observed, and the drop was proportional to hydrogen concentrations (Figure 4B). Within $\sim 30$ min the redox potential returned to baseline if the headspace was flushed with argon. The drop in redox potential observed upon hydrogen addition mimics the observation in MR-1 cultures when particulate Fe(III) oxide is the electron acceptor. 

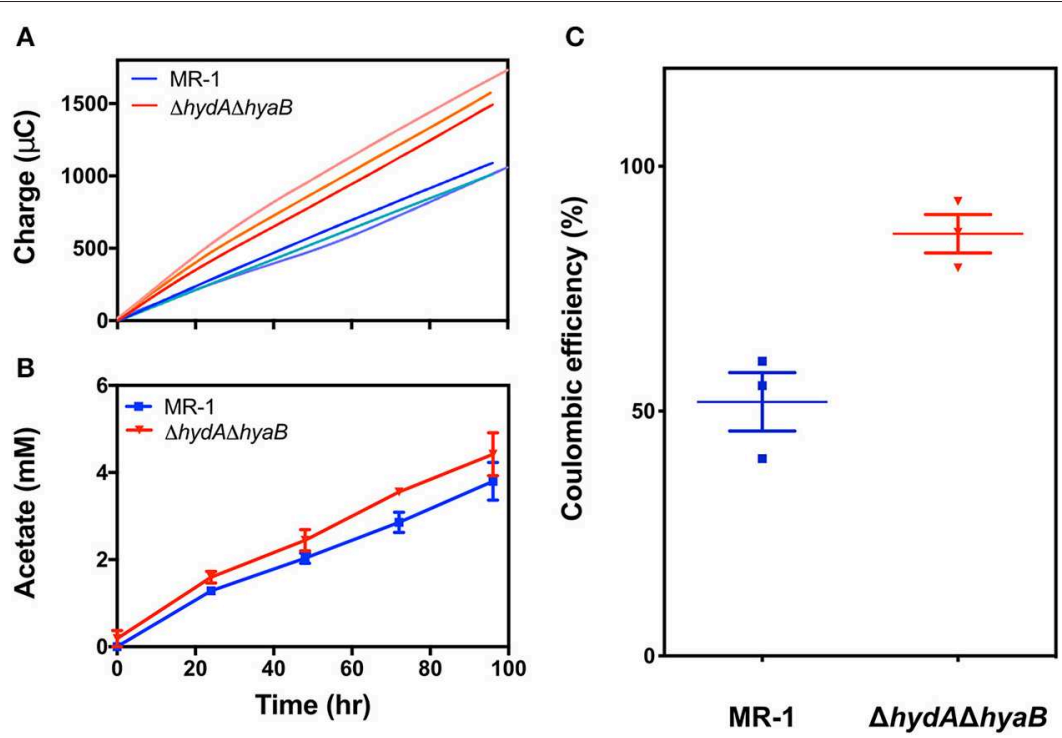

FIGURE 3 | Deletion of hydrogenases diverts electron flux toward the anode, increasing efficiency of electron recovery. (A) Recovered charge measured for reactors containing MR-1 (blue) as compared to $\triangle$ hydA $\triangle$ hyaB (red), reflecting the faster rate of current production by hydrogenase mutants. Each line represents one of three replicate reactors. (B) Acetate production rates for MR-1 (blue) and $\triangle$ hydA $\triangle$ hyaB (red), revealing similar rates of electron donor consumption, measured by HPLC in triplicate reactors. Error is represented as SEM. Time on the $\mathrm{x}$-axis reflects time since replacing the medium in reactors containing mature biofilms with fresh $30 \mathrm{mM}$ lactate and $1 \mu \mathrm{M}$ riboflavin. (C) Coulombic efficiency measured as mole equivalents of electrons transferred to the anode vs. mole equivalents of electrons produced by lactate oxidized to acetate in MR-1 (blue) and $\triangle$ hydA $\triangle$ hyaB (red) in reactors with vented cathodes. Three independent experiments are shown for each strain with error representing SEM.
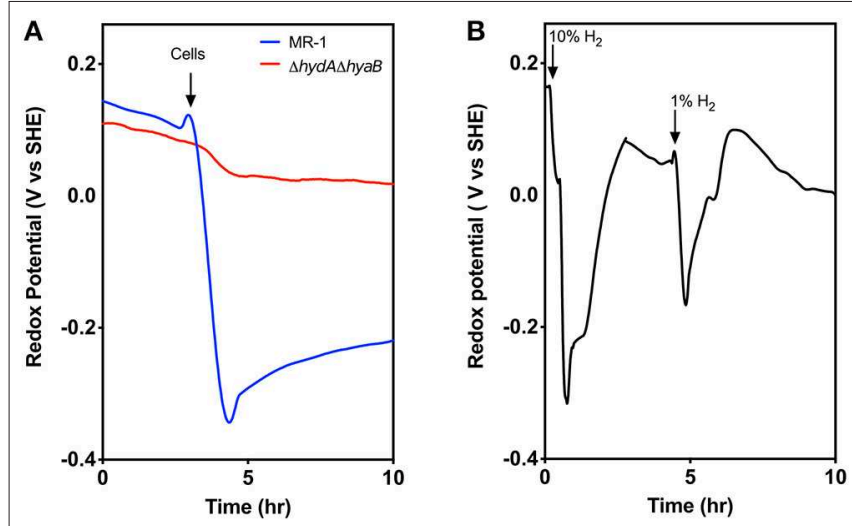

FIGURE 4 | Evidence for hydrogen disposal by MR-1 cells after transfer to $\mathrm{Fe}$ (III) oxide containing medium. (A) Redox probe based measurements detect a rapid decrease in redox potential when MR-1 cells with excess lactate are exposed to Fe(III) oxide as an electron acceptor. In contrast, $\triangle$ hydA $\Delta$ hyaB mutants do not lower redox potential, consistent with a burst of produced hydrogen by wild type cells experiencing donor-excess conditions. Fe(II) reduction at this early time point was minimal and similar by both strains (data not shown). (B) Abiotic control experiments showing similar rapid drop in redox potential in $\mathrm{Fe}$ (III) oxide medium in response to small additions of hydrogen to the headspace of reactors, and removal by flushing. Data is representative of three replicates.

\section{Hydrogen Production Enhances Stationary Phase Survival}

While deletion of hydrogenases diverted electrons to an electrode in a manner beneficial to the operation of a bioelectrochemical device, the presence of hydrogenases likely benefits the organism and aids survival under less steady-state conditions. To examine the effects of hydrogenases during starvation under conditions where cell viability could be easily determined, MR-1 and the $\Delta h y d A \triangle h y a B$ strain were grown with $\mathrm{Fe}(\mathrm{III})$ citrate as the sole terminal electron acceptor, which allowed the concentration of $\mathrm{Fe}$ (II) and cell density to be easily monitored over time. Reduction rates of $\mathrm{Fe}(\mathrm{III})$ citrate were not significantly different between MR-1 and the $\triangle h y d A \Delta h y a B$ strain (Figure 5A), and both reduced all available $\mathrm{Fe}$ (III) (Supplementary Figure 3). However, survival of MR-1 in stationary phase was greater than that of $\triangle h y d A \Delta h y a B$ (Figure $5 B$ ). A likely explanation for this phenotype is that disposal of hydrogen into the headspace by the wild type provided a route for a maintenance level of metabolism and enhanced survival.

\section{DISCUSSION}

Biochemically-derived hydrogen is generated during processes as diverse as fermentation and photosynthesis, is often a key molecule driving the thermodynamics of syntrophic partnerships (Stams and Plugge, 2009; Schink and Stams, 2013), and even affects virulence of Salmonella species in the gut (Spear et al., 2005; Maier et al., 2013). Particularly in anoxic environments, hydrogen concentrations are a controlling factor of microbial activity, and under steady state conditions can be used as an indicator of which terminal electron acceptor reactions are predominant (Lovley and Goodwin, 1988). The data presented here demonstrate how hydrogen is an important molecule in the ecophysiology of Shewanella, in particular within the engineered 

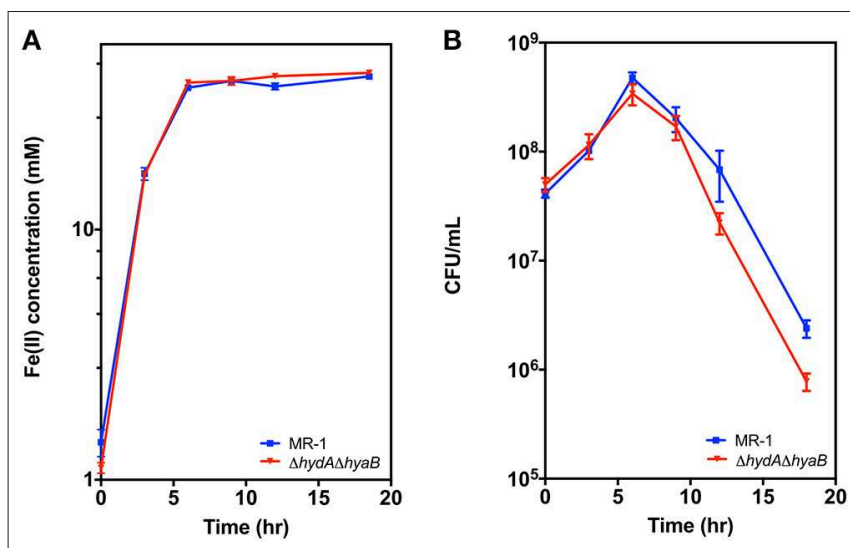

FIGURE 5 | Survival of MR-1 is enhanced compared to $\Delta$ hydA $\Delta$ hyaB. Strains were grown in vented bioreactors with $\mathrm{Fe}(\mathrm{III})$ citrate $(50 \mathrm{mM})$ as the terminal electron acceptor and lactate $(10 \mathrm{mM})$ as electron donor. Cultures were monitored over time for (A) Fe(II), and (B) cell density measured as colony forming units (CFU). Data represents three independent cultures with error reported as SEM.

environments of bioelectrochemical systems. Shewanella species thrive in a variety of environments because they encode multiple electron transport pathways enabling the use of a wide array of terminal electron acceptors, including protons, as they become available.

To study electron flux in Shewanella, it was essential to understand the fate of carbon sources and electron carriers as well as the efficiency of electron transfer to and from electrodes. Reported electron recovery calculations for MR-1 using a variety of reactor types in the literature range from 1.3$75 \%$, with the majority between 10-20\% (Ringeisen et al., 2006, 2007; Newton et al., 2009; Bretschger et al., 2010; Rosenbaum et al., 2010, 2011; Watson and Logan, 2010; Kouzuma et al., 2012; Wu et al., 2013; Kirchhofer et al., 2014; TerAvest and Angenent, 2014; Luo et al., 2016). Low recovery values indicate that most of the electrons generated from oxidation of lactate remained unaccounted for, even when the focus of the studies was electricity production. Transfer of electrons to oxygen due to oxygen-permeable materials can account for much of this electron loss. In our own experiments, even the simple act of flushing reactors with anaerobic gases can actually introduce oxygen to the system. For example, preliminary experiments conducted while redesigning reactors found that tubing that has been autoclaved multiple times becomes permeable enough to increase the $\mathrm{O}_{2}$ in a reactor, as does introduction of a plastic membrane filter in the gas line. If the headspace contains even $10 \mathrm{ppm} \mathrm{O}_{2}, S$. oneidensis cells respiring at rates shown in Figure 2 could easily divert $\sim 50 \%$ of lactate oxidation to aerobic respiration over a 7 -day incubation. The modified bioreactor system reported here was optimized to maintain oxygen levels below $2 \mathrm{ppm}$, and to minimize flushing of the headspace to limit delivery of further oxygen into the system.

Kozuma et al. attempted to limit oxygen losses by deleting the three terminal oxidases in MR-1, but the triple mutant was unable to produce current, likely due to oxygen toxicity caused by earlier reactor designs (Kouzuma et al., 2012). Higher coulombic efficiency (84 $\pm 7 \%$ ) has been reported for MR-1 exposed to the conjugated oligoelectrolyte $\mathrm{DSSN}^{+}$that intercalates in membranes (Kirchhofer et al., 2014), possibly via inhibition of hydrogenases. Here we report a coulombic efficiency of $86.2 \pm$ $6.8 \%$ in bioreactors through redesign of reactor components, and by diverting electron flux away from hydrogen production toward electrode reduction through deletion of the hydrogenase subunits hydA and hyaB, in S. oneidensis (Figure 3C).

Removing the hydrogenases from $S$. oneidensis shuts down a "leaky" pathway for electron disposal in electrochemical reactors, but we find evidence that this pathway may prove important in environments when terminal electron acceptors are limited. Conversion of protons to molecular hydrogen by hydrogenases is a relatively simple reaction, but it is limited by thermodynamic constraints due to its low standard redox potential $(-414 \mathrm{mV})$ (Thauer et al., 1977). As hydrogen concentrations rise, continued hydrogen production becomes thermodynamically unfavorable from standard electron carriers with more positive redox potentials such as NADH $(-320 \mathrm{mV})$ (Thauer et al., 1977; Stams and Plugge, 2009). Especially as the first step of lactate oxidation to pyruvate has a formal midpoint potential of $-190 \mathrm{mV}$, even small amounts of ambient hydrogen prevents complete electron disposal via this pathway.

The new reactor design and double mutant allowed us to show that when utilizing an electrode as the terminal electron acceptor, MR-1 normally diverts some electron flux toward molecular hydrogen production. The small population size on an electrode, and low hydrogen concentration in these reactors further eases the thermodynamic constraints on hydrogen production as an electron disposal mechanism. As reduction rates of $\mathrm{Fe}$ (III) citrate were similar for both strains, soluble $\mathrm{Fe}(\mathrm{III})$ doesn't create the same bottleneck to electron transfer as an insoluble substrate. However, the increased survival of MR-1 containing hydrogenases indicates that maintenance of hydrogenases in a population provides a competitive advantage, at least in stationary phase conditions. As a respiratory generalist, Shewanella have been selected to dispose of electrons via any means available. This allows them to grow in a variety of environments or participate in syntrophic partnerships that consume their produced hydrogen (Nealson and Scott, 2006; Hau and Gralnick, 2007). However, under the more defined conditions of electrochemical systems, it may be necessary to remove some of this respiratory flexibility, to direct electron flow to electrodes and increase the efficiency of electron capture.

\section{DATA AVAILABILITY}

The datasets generated for this study will not be made publicly available. There are no datasets.

\section{AUTHOR CONTRIBUTIONS}

$\mathrm{KJ}$ and $\mathrm{DB}$ designed the bioreactor described here. KJ, AK, $\mathrm{NK}$, JG, and DB designed experiments. KJ, AK, and NK performed experiments. 


\section{FUNDING}

This work was supported by the Office of Naval Research (Award \#N00014-12-1-0309 and \#N00014-13-1-0552 to JG and Award \#N00014-18-1-2632 to JG and DB).

\section{ACKNOWLEDGMENTS}

We would like to thank Geoff Harms (University of Minnesota) for help in constructing modified parts for bioreactors. This work

\section{REFERENCES}

Baron, D., LaBelle, E., Coursolle, D., Gralnick, J. A., and Bond, D. R. (2009). Electrochemical measurement of electron transfer kinetics by Shewanella oneidensis MR-1. J. Biol. Chem. 284, 28865-28873. doi: 10.1074/jbc.M109.043455

Beblawy, S., Bursac, T., Paquete, C., Louro, R., Clarke, T. A., and Gescher, J. (2018). Extracellular reduction of solid electron acceptors by Shewanella oneidensis. Mol. Microbiol. 109, 571-583. doi: 10.1111/mmi.14067

Bretschger, O., Cheung, A. C. M., Mansfeld, F., and Nealson, K. H. (2010). Comparative microbial fuel cell evaluations of Shewanella spp. Electroanalysis 22, 883-894. doi: 10.1002/elan.200800016

Bretschger, O., Obraztsova, A., Sturm, C. A., Chang, I. S., Gorby, Y. A., Reed, S. B., et al. (2007). Current production and metal oxide reduction by Shewanella oneidensis MR-1 wild type and mutants. Appl. Environ. Microbiol. 73, 7003-7012. doi: 10.1128/AEM.01087-07

Coursolle, D., Baron, D. B., Bond, D. R., and Gralnick, J. A. (2010). The Mtr respiratory pathway is essential for reducing flavins and electrodes in Shewanella oneidensis. J. Bacteriol. 192, 467-474. doi: 10.1128/JB.00925-09

Fredrickson, J. K., Romine, M. F., Beliaev, A. S., Auchtung, J. M., Driscoll, M. E., Gardner, T. S., et al. (2008). Towards environmental systems biology of Shewanella. Nat. Rev. Microbiol. 6, 592-603. doi: 10.1038/nrmicro1947

Geelhoed, J. S., and Stams, A. J. M. (2011). Electricity-assisted biological hydrogen production from acetate by Geobacter sulfurreducens. Environ. Sci. Technol. 45, 815-820. doi: 10.1021/es102842p

Golitsch, F., Bücking, C., and Gescher, J. (2013). Proof of principle for an engineered microbial biosensor based on Shewanella oneidensis outer membrane protein complexes. Biosens. Bioelectron. 47, 285-291. doi: 10.1016/j.bios.2013.03.010

Hau, H. H., Gilbert, A., Coursolle, D., and Gralnick, J. A. (2008). Mechanism and consequences of anaerobic respiration of cobalt by Shewanella oneidensis Strain MR-1. Appl. Environ. Microbiol. 74, 6880-6886. doi: 10.1128/AEM.00840-08

Hau, H. H., and Gralnick, J. A. (2007). Ecology and biotechnology of the genus Shewanella. Annu. Rev. Microbiol. 61, 237-258. doi: 10.1146/annurev.micro.61.080706.093257

Hunt, K. A., Flynn, J. M., Naranjo, B., Shikhare, I. D., and Gralnick, J. A. (2010). Substrate-level phosphorylation is the primary source of energy conservation during anaerobic respiration of Shewanella oneidensis strain MR-1. J. Bacteriol. 192, 3345-3351. doi: 10.1128/JB.00090-10

Kane, A. L., Brutinel, E. D., Joo, H., Maysonet, R., VanDrisse, C. M., Kotloski, N. J., et al. (2016). Formate metabolism in Shewanella oneidensis generates proton motive force and prevents growth without an electron acceptor. J. Bacteriol. 198, 1337-1346. doi: 10.1128/JB.00927-15

Kirchhofer, N. D., Chen, X., Marsili, E., Sumner, J. J., Dahlquist, F. W., and Bazan, G. C. (2014). The conjugated oligoelectrolyte DSSN+ enables exceptional coulombic efficiency via direct electron transfer for anode-respiring Shewanella oneidensis MR-1-a mechanistic study. Phys. Chem. Chem. Phys. 16, 20436-20443. doi: 10.1039/C4CP03197K

Kouzuma, A., Hashimoto, K., and Watanabe, K. (2012). Influences of aerobic respiration on current generation by Shewanella oneidensis MR-1 in singlechamber microbial fuel cells. Biosci. Biotechnol. Biochem. 76, 270-275. doi: $10.1271 /$ bbb.110633 is dedicated in fond memory of Ethan Johnson (1972-2010) who began these studies on hydrogenase mutants in S. oneidensis. We also thank members of the Gralnick and Bond Laboratories for helpful discussions throughout the project.

\section{SUPPLEMENTARY MATERIAL}

The Supplementary Material for this article can be found online at: https://www.frontiersin.org/articles/10.3389/fenrg. 2019.00095/full\#supplementary-material
Kreuzer, H. W., Hill, E. A., Moran, J. J., Bartholomew, R. A., Yang, H., and Hegg, E. L. (2014). Contributions of the [NiFe]- and [FeFe]-hydrogenase to $\mathrm{H}_{2}$ production in Shewanella oneidensis MR-1 as revealed by isotope ratio analysis of evolved $\mathrm{H}_{2}$. FEMS Microbiol. Lett. 352, 18-24. doi: 10.1111/1574-6968.12361

Lee, H.-S., and Rittmann, B. E. (2010). Significance of biological hydrogen oxidation in a continuous single-chamber microbial electrolysis cell. Environ. Sci. Technol. 44, 948-954. doi: 10.1021/es9025358

Lee, H.-S., Torres, C. I., Parameswaran, P., and Rittmann, B. E. (2009). Fate of $\mathrm{H}_{2}$ in an upflow single-chamber microbial electrolysis cell using a metal-catalyst-free cathode. Environ. Sci. Technol. 43, 7971-7976. doi: 10.1021/es900204j

Lovley, D. R., and Goodwin, S. (1988). Hydrogen concentrations as an indicator of the predominant terminal electron-accepting reactions in aquatic sediments. Geochim. Cosmochim. Acta 52, 2993-3003. doi: 10.1016/0016-7037(88)90163-9

Lovley, D. R., and Phillips, E. J. P. (1987). Rapid assay for microbially reducible ferric iron in aquatic sediments. Appl. Environ. Microbiol. 53, 1536-1540.

Lu, M., Chan, S., Babanova, S., and Bretschger, O. (2017). Effect of oxygen on the per-cell extracellular electron transfer rate of Shewanella oneidensis MR1 explored in bioelectrochemical systems. Biotechnol. Bioeng. 114, 96-105. doi: $10.1002 /$ bit. 26046

Luo, S., Guo, W., H., Nealson, K., and Feng, X., He, Z. (2016). ${ }^{13} \mathrm{C}$ pathway analysis for the role of formate in electricity generation by Shewanella Oneidensis MR-1 using lactate in microbial fuel cells. Sci. Rep. 6:20941. doi: 10.1038/srep20941

Maier, L., Vyas, R., Cordova, C. D., Lindsay, H., Schmidt, T. S. B., Brugiroux, S., et al. (2013). Microbiota-derived hydrogen fuels Salmonella typhimurium invasion of the gut ecosystem. Cell Host Microbe 14, 641-651. doi: 10.1016/j.chom.2013.11.002

Meshulam-Simon, G., Behrens, S., Choo, A. D., and Spormann, A. M. (2007). Hydrogen metabolism in Shewanella oneidensis MR-1. Appl. Environ. Microbiol. 73, 1153-1165. doi: 10.1128/AEM.01588-06

Nealson, K. H., and Scott, J. (2006). "Ecophysiology of the genus Shewanella," in The Prokaryotes, eds M. Dworkin, S. Falkow, E. Rosenberg, K.-H. Schleifer, and E. Stackebrandt (New York, NY: Springer), 1133-1151. doi: 10.1007/0-387-30746-X_45

Newton, G. J., Mori, S., Nakamura, R., Hashimoto, K., and Watanabe, K. (2009). Analyses of current-generating mechanisms of Shewanella loihica PV-4 and Shewanella oneidensis MR-1 in microbial fuel cells. Appl. Environ. Microbiol. 75, 7674-7681. doi: 10.1128/AEM.01142-09

Pinchuk, G. E., Geydebrekht, O. V., Hill, E. A., Reed, J. L., Konopka, A. E., Beliaev, A. S., et al. (2011). Pyruvate and lactate metabolism by Shewanella oneidensis MR-1 under fermentation, oxygen limitation, and fumarate respiration conditions. Appl. Environ. Microbiol. 77, 8234-8240. doi: 10.1128/AEM.05382-11

Rabaey, K., and Verstraete, W. (2005). Microbial fuel cells: novel biotechnology for energy generation. Trends Biotechnol. 23, 291-298. doi: 10.1016/j.tibtech.2005.04.008

Ringeisen, B. R., Henderson, E., Wu, P. K., Pietron, J., Ray, R., Little, B., et al. (2006). High power density from a miniature microbial fuel cell using Shewanella oneidensis DSP10. Environ. Sci. Technol. 40, 2629-2634. doi: 10.1021/es052254w

Ringeisen, B. R., Ray, R., and Little, B. (2007). A miniature microbial fuel cell operating with an aerobic anode chamber. J. Power Sources 165, 591-597. doi: 10.1016/j.jpowsour.2006.10.026 
Rodionov, D. A., Yang, C., Li, X., Rodionova, I. A., Wang, Y., Obraztsova, A. Y., et al. (2010). Genomic encyclopedia of sugar utilization pathways in the Shewanella genus. BMC Genomics 11:494. doi: 10.1186/1471-2164-11-494

Rosenbaum, M., Cotta, M. A., and Angenent, L. T. (2010). Aerated Shewanella oneidensis in continuously fed bioelectrochemical systems for power and hydrogen production. Biotechnol. Bioeng. 105, 880-888. doi: 10.1002/bit.22621

Rosenbaum, M. A., Bar, H. Y., Beg, Q. K., Segrè, D., Booth, J., Cotta, M. A., et al. (2011). Shewanella oneidensis in a lactate-fed pure-culture and a glucose-fed coculture with Lactococcus lactis with an electrode as electron acceptor. Bioresour. Technol. 102, 2623-2628. doi: 10.1016/j.biortech.2010.10.033

Saltikov, C. W., and Newman, D. K. (2003). Genetic identification of a respiratory arsenate reductase. Proc. Natl. Acad. Sci. U.S.A. 100, 10983-10988. doi: $10.1073 /$ pnas. 1834303100

Schink, B., and Stams, A. J. M. (2013). "Syntrophism among prokaryotes," in The Prokaryotes: Prokaryotic Communities and Ecophysiology, eds E. Rosenberg, E. F. DeLong, S. Lory, E. Stackebrandt, and F. Thompson (Berlin; Heidelberg: Springer Berlin Heidelberg), 471-493. doi: 10.1007/978-3-64230123-0_59

Spear, J. R., Walker, J. J., McCollom, T. M., and Pace, N. R. (2005). Hydrogen and bioenergetics in the Yellowstone geothermal ecosystem. Proc. Natl. Acad. Sci. U.S.A. 102, 2555-2560. doi: 10.1073/pnas.0409574102

Stams, A. J. M., and Plugge, C. M. (2009). Electron transfer in syntrophic communities of anaerobic bacteria and archaea. Nat. Rev. Microbiol. 7, 568-577. doi: $10.1038 /$ nrmicro2166

TerAvest, M. A., and Angenent, L. T. (2014). Oxidizing electrode potentials decrease current production and coulombic efficiency through cytochrome $c$ inactivation in Shewanella oneidensis MR-1. ChemElectroChem 1, 2000-2006. doi: 10.1002/celc.201402128
TerAvest, M. A., Rosenbaum, M. A., Kotloski, N. J., Gralnick, J. A., and Angenent, L. T. (2014). Oxygen allows Shewanella oneidensis MR-1 to overcome mediator washout in a continuously fed bioelectrochemical system. Biotechnol. Bioeng. 111, 692-699. doi: 10.1002/bit.25128

Thauer, R. K., Jungermann, K., and Decker, K. (1977). Energy conservation in chemotrophic anaerobic bacteria. Bacteriol. Rev. 41, 100-180.

Venkateswaran, K., Moser, D. P., Dollhopf, M. E., Lies, D. P., Saffarini, D. A., MacGregor, B. J., et al. (1999). Polyphasic taxonomy of the genus Shewanella and description of Shewanella oneidensis sp. nov. Int. J. Syst. Bacteriol. 49(Pt 2), 705-724. doi: 10.1099/00207713-49-2-705

Watson, V. J., and Logan, B. E. (2010). Power production in MFCs inoculated with Shewanella oneidensis MR-1 or mixed cultures. Biotechnol. Bioeng. 105, 489-498. doi: 10.1002/bit.22556

Wu, D., Xing, D., Lu, L., Wei, M., Liu, B., and Ren, N. (2013). Ferric iron enhances electricity generation by Shewanella oneidensis MR-1 in MFCs. Bioresour. Technol. 135, 630-634. doi: 10.1016/j.biortech.2012.09.106

Conflict of Interest Statement: The authors declare that the research was conducted in the absence of any commercial or financial relationships that could be construed as a potential conflict of interest.

Copyright $\odot 2019$ Joshi, Kane, Kotloski, Gralnick and Bond. This is an open-access article distributed under the terms of the Creative Commons Attribution License (CC $B Y)$. The use, distribution or reproduction in other forums is permitted, provided the original author(s) and the copyright owner(s) are credited and that the original publication in this journal is cited, in accordance with accepted academic practice. No use, distribution or reproduction is permitted which does not comply with these terms. 